\title{
Handling of Sore Noses: a Proof of Concept
}

\author{
Nadine Wurzer-Materna ${ }^{1} \cdot$ Uta Thieme $^{2} \cdot$ Dieter Werdier $^{3} \cdot$ Peter Meiser $^{4}$ (D)
}

Accepted: 26 March 2020 / Published online: 21 April 2020

(C) The Author(s) 2020

\begin{abstract}
Dryness and soreness of the anterior part of the nose frequently occur and hinder patients' quality of life. Nasal ointments are broadly applied in such cases, bringing about at least subjective improvement of clinical signs and symptoms. The mode of action of such treatments is occlusion of the wounded skin parts, which is commonly already achieved by the ointment base. However, the clinical benefit of such treatments has yet to be proven. In this trial, 100 patients suffering from soreness of the anterior part of the nose were randomized to receive either an anhydrous nasal ointment or no local treatment. Area under the curve built from daily average scores of the symptoms sensation of dryness, soreness, scaliness, and pain, evaluated by the patients on a daily basis over a period of 9 days, was the predefined primary endpoint of this trial. Symptoms were furthermore assessed by the physicians, and patients allocated to the treatment group assessed their perceptions on the use of the nasal ointment on the initiation and the close-out visit. Clinical signs and symptoms improved significantly between the initial and close-out visits in both ointment and control groups. However, treatment with the nasal ointment proved to be significantly superior to no therapy. Both evaluation of patients' perceptions of the treatment and tolerability assessment substantiate the safe and efficacious use of the tested nasal ointment.
\end{abstract}

Keywords Nasal ointment $\cdot$ Rhinitis $\cdot$ Rhinitis sicca $\cdot$ Wound healing

\section{Introduction}

Substance-based medical devices are considered "borderline" products with similar appearance and intended purpose as compared with medicinal products, but, by definition with a primarily non-pharmacologic mode of action. Classic examples are moistening nasal sprays or eye drops. Rising regulatory requirements, such as European Medical Device Regulation EU 2017/745 [1], entail efforts to provide stronger clinical evidence to support the safety and performance of the devices. We intended to substantiate the safety and performance of an established medical device nasal ointment that is used for the moistening and care of the anterior part of the

\section{This article is part of the Topical Collection on Medicine}

Peter Meiser

p.meiser@ursapharm.de

ENT praxis, Hofackerstraße 19, 86179 Augsburg, Germany

2 ENT praxis, Mercatorstraße 10-12, 47051 Duisburg, Germany

3 SAM GmbH, Tittardshang 5, 52072 Aachen, Germany

4 Ursapharm Arzneimittel GmbH, Industriestraße 35, 66129 Saarbrucken, Germany nose in the case of rhinitis sicca anterior and for the treatment of soreness due to acute or chronic rhinosinusitis, thus conditions commonly occurring in clinical practice. The device acts simply by occlusion of the wounded skin parts, which is commonly already achieved by the ointment base. Thus, we were confronted with the challenge of how to measure the effects resulting from this physical mode of action, since there is no placebo in the classic sense. We nevertheless succeeded in proving the effectiveness of the ointment and now present our approach in this brief communication.

\section{Methods}

This clinical trial was based on a prospective, randomized, two-armed longitudinal design with an initial and a final visit. Adults and children older than 12 years who had an indication for treatment with a regenerating nasal ointment on the anterior part of the nose (e.g., patients suffering from rhinitis sicca anterior, rhinosinusitis, and allergic rhinitis) were eligible for the study. Patients were excluded from participation in case of preceding local treatment, use of systemic antibiotics, in case of expected unreliability, and in case of concurrent or preceding participation in another trial (4 weeks before potential 
Table 1 Baseline characteristics of included patients given as number (sex) or mean ( \pm st.dev.)

\begin{tabular}{llll}
\hline & Nasal ointment & No treatment & $p$ value \\
\hline Sex (male/female) & $19 / 31$ & $15 / 35$ & $p>0.2$ \\
Age [years] & $41.1 \pm 14.3$ & $40.3 \pm 14.7$ & $p>0.2$ \\
Height [cm] & $172 \pm 10.3$ & $170.1 \pm 9.5$ & $p=0.093$ \\
Weight [kg] & $75.0 \pm 13.4$ & $77.0 \pm 17.7$ & $p>0.2$ \\
BMI [kg/m $\left.{ }^{2}\right]$ & $25.2 \pm 4.1$ & $26.6 \pm 5.7$ & $p=0.026$ \\
\hline
\end{tabular}

Sex distribution was tested by chi-square test; all other parameters were tested by two-sided $t$ tests for independent samples, $d f=98$; homogeneity is defined as $p>0.2$ [3]

inclusion into this trial). Patients were enrolled only after they had been given sufficient information and had provided written consent concerning their participation in the trial. The final visit was scheduled 10 days, maximally 12 days, after the initial examination. Patients were instructed to complete a patient diary on a daily basis. The study was carried out in 2 trial centers in Germany during the period October 2018March 2019.

The test product was an anhydrous ointment containing retinol palmitate, paraffin, liquid paraffin, wool fat, and white petrolatum. One hundred patients suffering from soreness of the anterior part of the nose were block randomized, after written informed consent, to receive either the ointment (to be applied thrice a day onto the affected skin area of the external nose as well as the anterior inner skin part of the nose) or no local treatment. Patients evaluated on a daily basis, by documentation in patient diaries, their sensation of dryness, soreness, scaliness, and pain on an ordinal scale of $0-4(0$, none; 1 , mild; 2 , moderate; 3 , strong; 4 , very strong) over a defined period of 9 days. Area under the curve (AUC) built from daily average scores of the four symptoms was the predefined primary endpoint of this trial. Sample size calculation was based on the primary endpoint: an effect of $d=0.7 \sigma$ was assumed to be clinically relevant, where $\sigma=\sigma$ test $=\sigma$ control is the standard deviation under the assumption of normal distributed random variables of each group. To detect an effect of $0.7 \sigma$ with an aimed power of $1-\beta=90 \%$ at a significance level of $\alpha=0.05,45$ patients per group are necessary for two-sided $t$ tests. Compensating for a lost-to-follow-up rate of $10 \%, 50$ patients per group were needed. During initial and final visit, dryness, erythema, soreness, and scaliness were also independently assessed by the physicians involved on an ordinal scale of $0-4$ (0, none; 1 , mild; 2 , moderate; 3 , strong; 4 , very strong). First application of the ointment took place on the initiation visit, and patients allocated to the treatment group assessed their perceptions (overall impression, irritation, odor sensation, nasal care, nasal moistening) on a numerical scale of $0-10(0$, worst grade; 10 , best grade) that was adapted from the recently introduced Nasal Spray Sensory Scale [2]. Irritation was tested initially since hypersensitivity reactions (e.g., itching, redness) against one of the ingredients cannot be ruled out. Odor sensation of the nasal ointment was also assessed only once, whereas overall impression, nasal care, and nasal moistening were also assessed during the close-out visit by the patients in order to further evaluate the effectiveness of the nasal ointment over the full period of application. During the final visit, physicians undertook a global evaluation on the efficacy of the treatment (very good - good - satisfactory - bad). Occurrence of any adverse event as well as an assessment of tolerability of the treatment ("flawless," "acceptable," or "not acceptable") was recorded by the physicians during the final visit. Baseline characteristics and safety evaluations were assessed on the safety population (all included patients). Primary and secondary endpoints were assessed on the intention-to-treat population. Sample size calculation and analyses were performed with SAS 9.3 (SAS Institute). The study was approved by the Ethic Committee of the North Rhine Medical Association and has been performed in accordance with the ethical standards as laid down in the 1964 Declaration of Helsinki and its later amendments and was registered in the German Clinical Trial Register (DRKS00015670).
Table 2 Diagnoses of included patients

\begin{tabular}{lcc}
\hline Indication & Nasal ointment & No treatment \\
\hline Rhinitis sicca anterior & 1 & 0 \\
Rhinitis sicca anterior and acute bacterial rhinosinusitis & 15 & 19 \\
Rhinitis sicca anterior and acute bacterial rhinosinusitis & 1 & 0 \\
$\quad$ and chronic rhinosinusitis & 12 & 7 \\
Rhinitis sicca anterior and acute viral rhinosinusitis & 2 & 0 \\
Allergic rhinitis & 0 & 1 \\
Allergic rhinitis and chronic rhinosinusitis & 4 & 4 \\
Acute bacterial rhinosinusitis & 15 & 19 \\
Acute viral rhinosinusitis & & \\
\hline
\end{tabular}


Fig. 1 Daily mean AUCs of clinical symptoms (sensation of dryness, soreness, scaliness, pain)
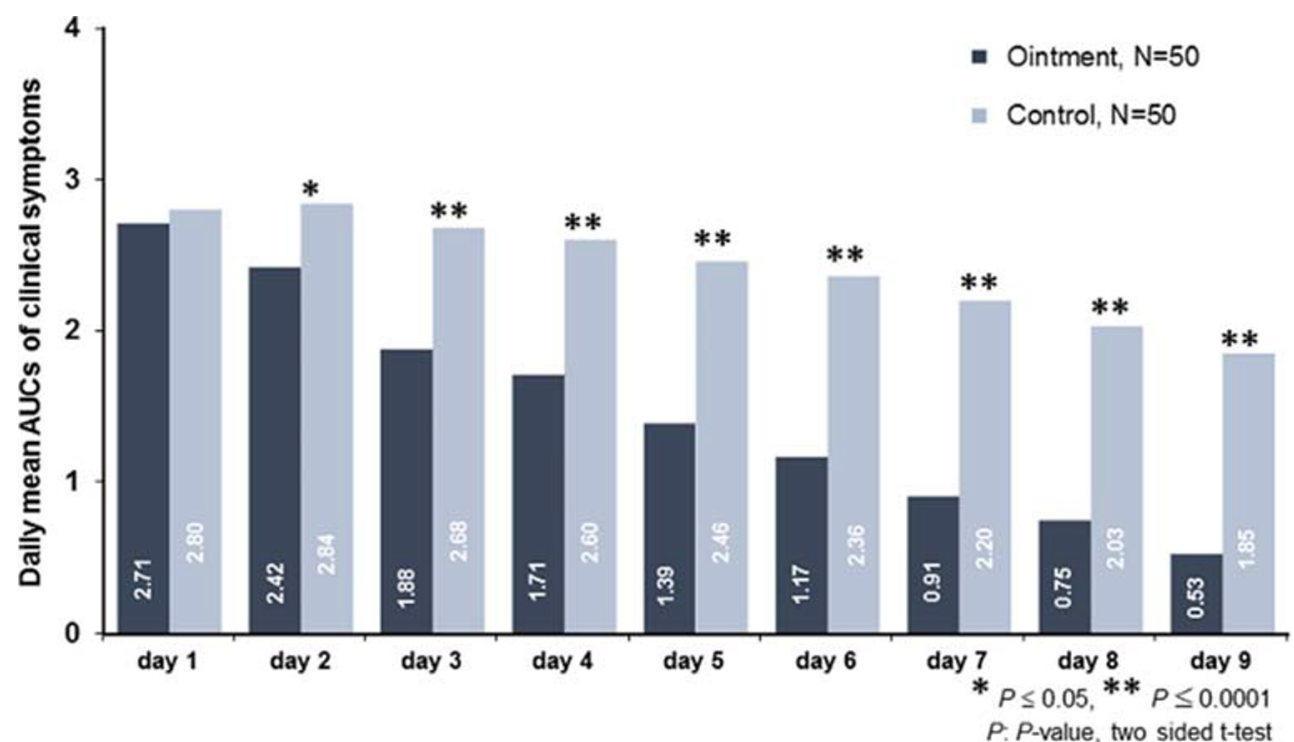

\section{Results}

Demographic data of included patients are given in Table 1, and patients' diagnoses are given in Table 2. Differences were observed for height and BMI between both study groups but are not considered to be of clinical relevance for the parameters tested within this study setting.

Individual clinical signs and symptoms, evaluated by patients and physicians, improved significantly between the initial and close-out visits in both ointment and control groups. Analysis of the primary parameter revealed a significant superiority of therapy vs. no therapy (AUC (ointment): $13.46 \pm$ 5.81 vs. AUC (control): $21.80 \pm 5.57 ; p<0.0001$ ). Figure 1 demonstrates the superiority of therapy, with differences becoming meaningful already on the second day of treatment. Evaluation of dryness, erythema, soreness, and scaliness by the involved physicians on the initial vs. the final visit revealed significant improvements in both study groups. However, improvement of symptoms was significantly superior under therapy vs. no therapy $(p<0.0001)$. Perceptions of the treatment with the nasal ointment immediately after first application and at the end of the study are given in Table 3 . During the final visit, physicians evaluated the treatment as very good in $88 \%$ of the patients, as good in $10 \%$, and as satisfactory in $2 \%$ of the patients. Tolerability of treatment was assessed as "flawless" in 49 patients and as "acceptable" in one patient by the physicians. One adverse event (condition aggravated) occurred in a patient from the control group, and no adverse events were observed in the nasal ointment group.

\section{Discussion}

Dryness and soreness of the anterior part of the nose frequently occur in patients suffering from rhinitis sicca anterior and acute or chronic upper respiratory tract infections [4]. These symptoms are self-limiting but still vastly hinder patients' quality of life. Occlusive ointments promise improvement and are in broad clinical use. But, how can a clinical benefit be shown, considering that it is accepted knowledge that the ointment base itself already brings about the effect? Previous studies reported on the benefits of treatments with nasal
Table 3 Patients' perception (absolute numbers) of nasal ointment after first application (1st appl.) and at the end of the study period (close-out-visit); 0 , worst grade; 10, best grade

\begin{tabular}{llllllllllllll}
\hline & 0 & 1 & 2 & 3 & 4 & 5 & 6 & 7 & 8 & 9 & 10 & $p$ values \\
\hline Overall impression 1st appl. & 1 & - & - & - & - & 1 & 2 & 4 & 17 & 17 & 8 & $p<0.0001$ \\
Overall impression close-out visit & - & - & - & - & - & 1 & 2 & 2 & 6 & 14 & 25 & \\
Nasal care 1 1st appl. & - & - & - & - & - & 2 & 4 & 4 & 13 & 17 & 10 & $p<0.0001$ \\
Nasal care close-out visit & - & - & - & - & - & 1 & - & 3 & 7 & 14 & 25 & \\
Nasal moistening 1st appl. & 1 & - & - & - & - & 3 & 2 & 3 & 10 & 8 & 23 & $p=0.045$ \\
Nasal moistening close-out visit & - & - & - & - & - & 2 & 1 & 1 & 6 & 7 & 33 & \\
Irritation 1st appl. $_{\text {Odor sensation 1st appl. }}$ & - & - & - & - & - & - & - & 2 & 2 & 11 & 35 & \\
\hline
\end{tabular}

Differences were tested for by chi-square test 
ointments in patients suffering from allergic rhinitis [5-7]. In contrast, we included patients with various underlying diseases in our trial in order to obtain a broad overview on the clinical benefit of the nasal ointment. By way of testing the treatment with an occluding anhydrous nasal ointment against no local treatment, we indeed observed amelioration of symptoms in both study groups. Application of the nasal ointment resulted in significantly superior improvements, however, and confirms the clinical benefit of such measures. Our study thus demonstrates that patient diaries combined with nasal sensory scales are suitable tools for the measurement of effects in such "soft" indications. Besides the observed overall superiority of treatment with the nasal ointment vs. no treatment, as depicted in Fig. 1, another aspect of our results warrants discussion: patients reported a strong moistening effect (66\% of the patients treated with the ointment assigned the highest rating at the end of the study period) besides the application of an anhydrous nasal ointment. This observation clearly supports the intrinsic moistening of the affected skin due to the occlusive effect.

We only assessed the irritative potential of the nasal ointment once after the first application (refer to Table 3); however, absence of the occurrence of any adverse events and the observed good tolerability of the therapy demonstrates the favorable safety and tolerability profile of the nasal ointment.

\section{Conclusions}

Both evaluation of patients' perceptions of the treatment and tolerability assessment substantiate the safe and efficacious use of the tested nasal ointment.

Funding Information The trial was funded by URSAPHARM Arzneimittel GmbH. Peter Meiser is employed at URSAPHARM Arzneimittel $\mathrm{GmbH}$.

\section{Compliance with Ethical Standards}

Conflict of Interest The authors declared that they have no conflict of interest.

Ethical Approval All procedures performed in this study involving human participants were in accordance with the ethical standards of the institutional research committee and with the 1964 Helsinki declaration and its later amendments.

Informed Consent Informed consent was obtained from all individual participants included in the study.

Open Access This article is licensed under a Creative Commons Attribution 4.0 International License, which permits use, sharing, adaptation, distribution and reproduction in any medium or format, as long as you give appropriate credit to the original author(s) and the source, provide a link to the Creative Commons licence, and indicate if changes were made. The images or other third party material in this article are included in the article's Creative Commons licence, unless indicated otherwise in a credit line to the material. If material is not included in the article's Creative Commons licence and your intended use is not permitted by statutory regulation or exceeds the permitted use, you will need to obtain permission directly from the copyright holder. To view a copy of this licence, visit http://creativecommons.org/licenses/by/4.0/.

\section{References}

1. Regulation (EU) $2017 / 745$ of the European Parliament and of the Council of 5 April 2017 on medical devices, amending Directive 2001/83/EC, Regulation (EC) No 178/2002 and Regulation (EC) No 1223/2009 and repealing Council Directives 90/385/EEC and 93/42/EEC. Official Journal of the European Union. 2017;L177/1.

2. Mosges R, Pasch N, Sayar A, Schmalz P, Vent J. Survey of sensory perception and patients' subjective assessment of the application of nasal sprays - the nasal-spray-sensoric-scale. Laryngorhinootologie. 2009;88(9):587-91. https://doi.org/10.1055/s-0029-1202369.

3. Armitage P, Berry G, Matthews J. Statistical methods in medical research. 1st ed. New York: Wiley; 1971.

4. Hildenbrand T, Weber RK, Brehmer D. Rhinitis sicca, dry nose and atrophic rhinitis: a review of the literature. Eur Arch Otorhinolaryngol. 2011;268(1):17-26.

5. Bufe A. A simple advice for the prevention of pollen-induced allergic rhinitis. Int Arch Allergy Immunol. 2000;121(1):85-6.

6. Li Y, Cheng L, Chen X, Yang B, Wang D. Efficacy evaluation of a pollen blocker cream against dust-mite allergy: a multicenter, randomized, double-blind, placebo-controlled crossover trial. Am J Rhinol Allergy. 2015;29(5):e129-33.

7. Geisthoff UW, Blum A, Rupp-Classen M, Plinkert PK. Lipid-based nose ointment for allergic rhinitis. Otolaryngol Head Neck Surg. 2005;133(5):754-61.

Publisher's Note Springer Nature remains neutral with regard to jurisdictional claims in published maps and institutional affiliations. 